\title{
Comparison of Radiographic Progression-Free Survival and PSA Response on Sequential Treatment Using Abiraterone and Enzalutamide for Newly Diagnosed Castration-Resistant Prostate Cancer: A Propensity Score Matched Analysis from Multicenter Cohort
}

\author{
Kazumasa Komura ${ }^{1,2, *}$, Yuya Fujiwara ${ }^{1}$, Taizo Uchimoto ${ }^{3}$, Kenkichi Saito ${ }^{1}$, Naoki Tanda ${ }^{1}$, \\ Tomohisa Matsunaga ${ }^{4}$, Atsushi Ichihashi ${ }^{5}$, Takeshi Tsutsumi ${ }^{1}$, Takuya Tsujino ${ }^{6}{ }^{(1)}$, \\ Yuki Yoshikawa ${ }^{1}$, Yudai Nishimoto ${ }^{7}$, Tomoaki Takai ${ }^{6}{ }^{\circledR}$, Koichiro Minami $^{4}$, \\ Kohei Taniguchi ${ }^{2}{ }^{\mathbb{D}}$, Tomohito Tanaka ${ }^{2}$, Hirofumi Uehara ${ }^{1}$, Hajime Hirano ${ }^{1}$, Hayahito Nomi ${ }^{1}$, \\ Naokazu Ibuki ${ }^{1}$, Kiyoshi Takahara ${ }^{8}$, Teruo Inamoto ${ }^{1}$ and Haruhito Azuma ${ }^{1}$ \\ Department of Urology, Osaka Medical College, Osaka 569-8686, Japan \\ Translational Research Program, Osaka Medical College, Osaka 569-8686, Japan \\ Department of Urology, Saiseikai-Nakatsu Hospital, Osaka 530-0012, Japan \\ Department of Urology, Osaka Medical College Mishima-Minami Hospital, Osaka 569-0856, Japan \\ Department of Urology, Aijinkai-Takatsuki Hospital, Osaka 569-1192, Japan \\ Division of Urology, Department of Surgery, Brigham and Women's Hospital, Boston, MA 02115, USA \\ Department of Urology, Hirakata Municipal Hospital, Osaka 573-1013, Japan \\ Department of Urology, Fujita-Health University School of Medicine, Nagoya 470-1192, Japan \\ * Correspondence: uro051@osaka-med.ac.jp; Tel.: +81-726-83-1221
}

Received: 28 June 2019; Accepted: 16 August 2019; Published: 19 August 2019

\begin{abstract}
Background: There is emerging evidence that radiographic progression-free survival (rPFS) is highly correlated with overall survival (OS), potentially serving as an indicator of treatment outcome for castration-resistant prostate cancer (CRPC). The objective of this study is to assess rPFS and prostate specific antigen (PSA) response in sequential treatment using androgen signaling inhibitors (ASIs) including abiraterone and enzalutamide in newly diagnosed CRPC. Methods: Propensity score matching was performed to reduce bias by confounding factors between first-line ASIs. The primary endpoints of the study included rPFS, time to PSA progression (TTPP), and PSA response. Results: A paired-matched group of 184 patients were identified. From the initiation of first-line ASIs, there was no significant difference in rPFS, TTPP, and PSA response between treatment arms. From the initiation of second-line ASIs, enzalutamide following abiraterone consistently exhibited longer rPFS (median: 7 and 15 months, $p=0.04$ ), TTPP, and better PSA response compared to the reverse, whereas OS did not reach significance (median: 14 and 23 months, $p=0.35$ ). Conclusion: Although the effect of ASIs as the first line was similar, the extent of cross-resistance might differ towards less resistance in enzalutamide following abiraterone than the reverse.
\end{abstract}

Keywords: castration-resistant prostate cancer; abiraterone; enzalutamide; propensity score matched analysis; radiographic progression-free survival; sequential treatment

\section{Introduction}

Prostate cancer is one of the most common malignancies in men [1]. Although androgen deprivation therapy (ADT) offers certain remissions lasting 1 to 2 years in most patients, cancer cells 
eventually develop castration-resistant prostate cancer (CRPC) through multiple mechanisms [2-5]. Over the past decade, several new agents have been approved for the treatment of CRPC including two androgen signaling inhibitors (ASIs), namely, abiraterone acetate plus prednisone (referred to from here on as 'abiraterone') and enzalutamide. Following the first approval of these ASIs for the treatment of CRPC previously treated with docetaxel [6,7], these agents have been further approved in chemotherapy-naïve settings [8,9], even with the initiation of ADT $[10,11]$. As of now, these ASIs have been widely used due to their durable efficacy, convenient oral administration, and favorable toxicity profiles in real-world practice [12,13].

There have been a myriad of retrospective studies reporting the sequential use of ASIs for the treatment of CRPC - namely 'abiraterone following enzalutamide' and 'enzalutamide following abiraterone' [14-26]. The data from these results seems to indicate cross-resistance between these ASIs. In particular, a modest response of abiraterone after progression on docetaxel and enzalutamide was observed in patients after discontinuation from the AFFIRM trial [7], in which less than 10\% of patients achieved a $\geq 50 \%$ decline of prostate specific antigen (PSA) with subsequent abiraterone $[18,21]$. In 2017, the result of a phase 4, single-arm study (ClinicalTrials. Gov, NCT02116582) of enzalutamide in patients who had progressive disease following prior abiraterone treatment was reported from a multi-institutional collaboration in Europe [27]. This revealed that, despite the cross-resistance between these ASIs, enzalutamide following abiraterone appears to be active in CRPC with or without prior chemotherapy. In addition, most recently, the results from a phase 2 randomized cross-over trial (ClinicalTrials. Gov, NCT02125357) using these ASIs [28] exhibited that PSA response and time to PSA progression (TTPP) on second-line ASIs were significantly associated with favorable outcomes in patients treated with enzalutamide following abiraterone.

Several post-hoc studies from the results of randomized control trials (RCTs) revealed emerging evidence that radiographic progression-free survival (rPFS) is highly correlated with overall survival (OS), potentially serving as an indicator of treatment outcome in CRPC patients [29-31]. In 2015, Morris et al. first reported that the Spearman's correlation coefficient (SCC) between rPFS and OS was 0.71 (95\%CI: 0.65-0.77) in pot-hoc analysis from COU-AA-302 study (abiraterone vs placebo for chemotherapy-naïve metastatic CRPC) [30]. Subsequently, Rathkopf et al. showed similar SCC between rPFS and OS (SCC: $0.72,95 \%$ CI: 0.67-0.76) from the PREVAIL study (enzalutamide vs placebo for chemotherapy-naïve metastatic CRPC) [29]. Recently, the results from a prospective multicenter observational cohort study in 406 metastatic CRPC patients treated with ASIs or docetaxel as a first line (NCT03075735) demonstrated Pearson's correlation with OS of 0.65 in rPFS and 0.54 in TTPP [31]. In the present study, we conducted propensity score-matched analysis for the treatment of newly diagnosed CRPC patients treated with sequential ASIs from the first line, and assessed rPFS as well as PSA response in each line to investigate the extent of cross-resistance with these ASIs and the impacts on their prognosis.

\section{Materials and Methods}

This multicenter cohort study was designated to assess the treatment outcome of sequential therapy using abiraterone acetate (1000 $\mathrm{mg}$ ) plus prednisone (5 $\mathrm{mg}$ twice daily) and enzalutamide $(160 \mathrm{mg})$ in patients with newly diagnosed CRPC. The study design was approved by the institutional review board (IRB approval number: RIN-750-2571) and performed in accordance with the ethical standards of the World Medical Association Declaration of Helsinki [32]. CRPC was diagnosed by a serum testosterone level of $<50 \mathrm{ng} / \mathrm{dL}$, and either PSA progression (an increase of $25 \%$ and an absolute increase of $2 \mathrm{ng} / \mathrm{mL}$ or more above the PSA nadir) or radiographic progression (defined by PCWG2 guidelines) [33]. According to the previous reports showing a marginal survival benefit for CRPC remaining on LHRH analogs during subsequent therapies [34,35], all the patients indefinitely underwent LHRH analogs using ASIs with the treatment. In the present study, patients treated with ASIs for hormone-naïve prostate cancer were excluded. 
Figure 1 represents a scheme of the study in which a total of 357 CRPC patient records were collected, followed by a logistic regression propensity score model stratified by the type of first-line ASIs including abiraterone and enzalutamide. To estimate the propensity score (conditional probability), the following variables were included in the regression model: age at diagnosis (continuous variable), PSA at diagnosis (continuous variable), Gleason's sum grade $(<7,8,9,10)$, skeletal-related events (SRE) during follow-up $(-,+)$, ADT response duration $(<12$ months or not), taxane usage during follow-up $(-,+)$, bone metastasis at first-line treatment $(0,1,2,>3)$, LN metastasis at first-line treatment (none, pelvic, other sites), and visceral metastasis at first-line treatment $(-,+)$. Missing values were included in the model as separate categories as missing data may systematically differ between the two treatment groups. A 1:1 matching (without replacement) across the two treatment groups was achieved by the nearest neighbor method with a 0.2 -width caliper of the standard deviation of the logit of the propensity scores to reduce bias by those potential confounding factors. Matching was carried out using the JMP Pro Add-In package version 13.0.0 (SAS Institute Inc., Cary, NC, USA).

The primary endpoints of the study included rPFS, time to PSA progression (TTPP), and PSA response. The secondary endpoint involved overall survival (OS). Radiographic progression was evaluated based on PCWG2 guidelines [33]. As specified, development or progression of lymph nodes greater than $2 \mathrm{~cm}$ by spiral computed tomography (CT), as well as any other visceral or soft tissue lesions, were measured according to RECIST guidelines (ver1.1) [36]. For bone metastasis, radiological progression was defined as when at least two or more new lesions are seen on a bone scan compared with a prior scan. In cases where the scan findings are ambiguous (i.e., suggestive of a flare reaction or a trauma), confirmation by other imaging modalities such as MRI, fine-cut CT, and positron emission tomography (PET/CT) were required, which is not necessary if multiple new areas of uptake are observed [37]. Serum PSA level was measured every month from the baseline assessment at the initiation of the first-line treatment. PSA response was defined as nadir level in 6 months divided by the baseline PSA level. PSA progression, also referred to as TTPP, was also defined based on the PCWG2 guideline [33]. Briefly, an increase of $25 \%$ and an absolute increase of $2 \mathrm{ng} / \mathrm{mL}$ or more above the PSA nadir was considered PSA progression.

Clinical stages in each patient were evaluated by magnetic resonance imaging (MRI), computed tomography (CT), ultrasound, and chest-X rays, and other patient information including performance status (Eastern Cooperative Oncology Group, ECOG-PS) and all the clinical laboratory measurement in peripheral blood (CRP, albumin, alkaline phosphatase, lactate dehydrogenase, neutrophils, lymphocytes, platelets, and hemoglobin) were recorded at an initiation of first-line treatment. Follow-up $\mathrm{CT}$ to detect any findings suspected of disease progression were scheduled every 3 months from the diagnosis of CRPC. Based on the PCWG2 guidelines [33], MRI, bone scintigraphy, and PET/CT were further performed when necessary for a definitive diagnosis of disease progression. Follow-up was calculated from the day of initiation of first-line treatment to the day of death or final visit.

Clinicopathological findings in the analysis included patient age, PSA value, skeletal-related events (SRE), ECOG-PS, Gleason's sum score, and site of metastasis. The distribution of each factor was assessed by a contingency table with a Chi-square analysis. Kolmogorov-Smirnov normality was examined to check normal distribution in continuous variables, followed by a Student's $t$-test or one-way ANOVA to assess the difference between the variables. For variables with non-normal distribution, a Wilcoxon or Kruskal-Wallis test was performed to assess the difference. A Kaplan-Meier analysis was carried out to estimate the survival free ratio, and a log-rank test was performed to compare the differences between assigned patient groups. On univariate and multivariate analysis, Cox proportional-hazard regression models were used to estimate crude hazard ratios (HR) followed by calculating covariate-adjusted HR. All statistical tests were two-sided, with $p<0.05$ considered to indicate statistical significance. All analyses were performed using JMP Pro version 13.0.0 (SAS Institute Inc., Cary, NC, USA). 


\section{Results}

Figure 1 represents the study design for comparing the treatment outcome of abiraterone and enzalutamide from the first-line treatment. Of all 357 CRPC patients in the cohort, 242 patients were treated with either abiraterone (113 patients) or enzalutamide (129 patients) as the first-line treatment. To reduce any bias due to potential confounders that could affect treatment outcome between both arms, propensity score matching was performed using putative variables, as shown in Figure 1, from which 92 patients in each arm were identified as pair-matched groups. Table 1 exhibits that all the variables in the pair-matched groups had no significant differences between treatment arms. In total, $55(29.9 \%)$ patients deceased during their follow-up, and the median follow-up time for patients living and dead were 17 and 13 months, respectively. Of note, the matched cohort included 57 of M0 CRPC patients at the initiation of the first-line treatment. Two-year OS rates in M0 and M1 CRPC patients from initiation of the first-line treatment were $74.3 \%$ and $55.7 \%$, respectively $(p=0.03)$. Figure 2 illustrates rPFS, TTPP, and OS from the time of initiation of the first line according to the treatment. Kaplan-Meier curves showed no significant difference in rPFS, TTPP, and OS between abiraterone and enzalutamide from the first-line treatment. The median time to treatment failure was 12 months in abiraterone and 15 months in enzalutamide, with no significant difference between the arms $(p=0.30)$.

To further examine the effect of ASIs in sequential usage, we conducted subgroup analysis for patients who were treated with second-line ASIs-namely, either abiraterone following enzalutamide, or vice versa. There were 84 out of 184 patients who had subsequent second-line ASIs, whereas 20 patients were treated with taxanes as a second line. Comparing clinical characteristics between 'abiraterone to enzalutamide in 46 patients' and 'enzalutamide to abiraterone in 38 patients' showed similar patient backgrounds except for the PSA decline of more than $50 \%$ with the first-line ASIs (Table 2). In short, patients treated with 'enzalutamide following abiraterone' were less likely to achieve a PSA decline of $\geq 50 \%$ from the baseline at their first-line treatment compared to 'abiraterone following enzalutamide' ( $p=0.037$ ). With regard to PSA kinetics throughout the sequential treatment using these ASIs, we separately assessed PSA response in the first and second line (Figure 3). In the first-line treatment, PSA decline of more than 50\% from the baseline was observed in 59.3\% (54 of 92) for abiraterone and 67\% (60 of 92) for enzalutamide, with no significant difference (Chi-square: $p=0.28$ ). A Mann-Whitney test to assess PSA response between ASIs as the first line also exhibited no significant difference. To interrogate cross-resistance across these ASIs, we next investigated PSA response on second-line ASIs (i.e., the effect of enzalutamide following abiraterone and vice versa). Waterfall plots exhibited that PSA decline of more than 50\% from the initiation of second-line treatment was significantly less observed in abiraterone as a second line (8.3\%) compared to enzalutamide following abiraterone $(26.7 \%)(p=0.03)$. PSA response between these ASIs as the second line also exhibited better PSA response in enzalutamide following abiraterone than for the reverse $(p=0.01)$. TTPP from the initiation of the second line illustrated a significantly shorter TTPP in abiraterone as a second line (median: 3 months) compared to enzalutamide (median: 6 months), consistently indicating an attenuated effect on PSA in abiraterone as a second line after enzalutamide compared with the reverse (HR: 0.52, 95\%PI: 0.30-0.91, $p=0.008$ ) (Figure 4). Most importantly, rPFS from the initiation of the second line revealed that enzalutamide following abiraterone was significantly associated with a longer rPFS (median of 15 months) compared to abiraterone following enzalutamide (median of 7 months) (HR: 0.49 , 95\%CI: 0.25-0.98, $p=0.04$ ). Median OS from the initiation of the second line was 14 months in patients with 'abiraterone following enzalutamide', and 23 months with 'enzalutamide following abiraterone', which did not achieve a significant difference (HR: 0.76, 95\%CI: 0.41-1.41, $p=0.35$ ). 
CRPC Cohort Dataset

$(n=357)$

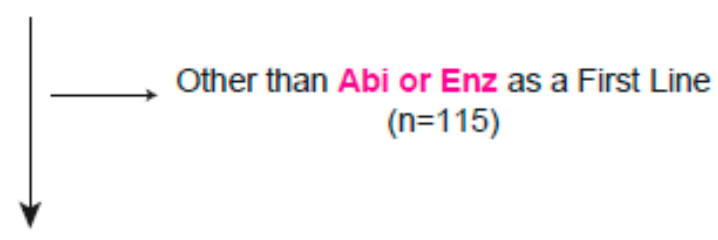

Abi or Enz as a First Line

$(n=242)$

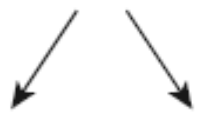

Abi as a First Line

$(n=113)$

Enz as a First Line

$(n=129)$

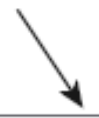

\section{Propensity Score Matching by}

- Age at diagnosis (continuous variable)

- PSA as diagnosis (continuous variable)

- Gleason' s sum grade $(\leq 7,8,9,10)$

- $\operatorname{SRE}(-,+)$

- ADT response duration (<12 months or not)

- Taxanes usage during follow-up $(-,+)$

- Bone mets at first line treatment $(0,1,2, \geq 3)$

- LN mets at first line treatment (non, pelvic, other sites)

- Visceral mets at first line treatment $(-,+)$

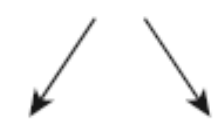

Abi as a First Line

$(n=92)$

Enz as a First Line

$(\mathrm{n}=92)$

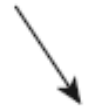

Primary Endpoint

Radiographic-Progression Free Survival (rPFS)

Time to PSA Progression (TTPP)

PSA Response Rate

Subgroup Analysis: (Abi to Enza) vs (Enz to Abi)

Radiographic-Progression Free Survival (rPFS)

Time to PSA Progression (TTPP)

PSA Response Rate

(Secondary Endpoint)

Figure 1. Study design and inclusion criteria of the propensity score-matched analysis in patients newly diagnosed with castration-resistant prostate cancer (CRPC). A 1:1 matching across the two treatment arms was performed using the nearest neighbor method with a 0.2 -width caliper of the standard deviation of the logit of the propensity scores. 
Table 1. Clinical characteristics in 184 CRPC patients adjusted by propensity score matching.

\begin{tabular}{|c|c|c|c|c|}
\hline Variables & Total $(n=184)$ & Abi $(n=92)$ & $\operatorname{Enz}(n=92)$ & $p$ Value \\
\hline Age (mean \pm SD) & $73.5+7.8$ & $74.0+8.0$ & $73.0+7.6$ & 0.355 \\
\hline \multicolumn{5}{|l|}{ SRE during follow-up } \\
\hline No $(\%)$ & $144(78.3)$ & $72(78.3)$ & $72(78.3)$ & \\
\hline Yes (\%) & $40(21.7)$ & $20(21.7)$ & $20(21.7)$ & 1.000 \\
\hline \multicolumn{5}{|l|}{ Taxanes during follow-up } \\
\hline No $(\%)$ & $159(86.4)$ & $80(87.0)$ & $79(85.9)$ & \\
\hline Yes (\%) & $25(13.6)$ & $12(13.0)$ & $13(14.1)$ & 0.809 \\
\hline \multicolumn{5}{|l|}{ ADT response duration } \\
\hline$\geq 12$ months $(\%)$ & $136(73.9)$ & $65(70.6)$ & $71(77.2)$ & \\
\hline$<12$ months $(\%)$ & $48(26.1)$ & $27(29.4)$ & $21(22.8)$ & 0.809 \\
\hline Median PSA level at diagnosis (ng/mL) (quartile) & $\begin{array}{l}124.0(29.3 \\
395.6)\end{array}$ & $\begin{array}{l}124.3(41.9 \\
327.1)\end{array}$ & $\begin{array}{l}93.8(25.7 \\
574.8)\end{array}$ & 0.685 \\
\hline $\begin{array}{c}\text { Median PSA level at first line treatment (ng/mL) } \\
\text { (quartile) }\end{array}$ & $6.8(2.3,30.1)$ & $6.8(2.0,30.4)$ & $6.8(2.5,30.1)$ & 0.918 \\
\hline \multicolumn{5}{|l|}{ Gleason sum score $(\%)$} \\
\hline$\leq 7$ & $20(10.9)$ & $9(9.8)$ & $11(12.0)$ & \\
\hline 8 & $41(22.3)$ & $20(21.7)$ & $21(22.8)$ & \\
\hline 9 & $113(61.4)$ & $56(60.9)$ & $57(62.0)$ & \\
\hline 10 & $10(5.4)$ & $7(7.6)$ & $3(3.3)$ & 0.598 \\
\hline \multicolumn{5}{|l|}{ Local treatment prior to ADT (\%) } \\
\hline Non & $156(84.8)$ & $77(83.7)$ & $79(85.9)$ & \\
\hline Prostatectomy & $16(8.7)$ & $9(9.8)$ & $7(7.6)$ & \\
\hline Radiation & $8(4.3)$ & $5(5.4)$ & $3(3.3)$ & \\
\hline Others & $4(2.2)$ & $1(1.1)$ & $3(3.3)$ & 0.311 \\
\hline \multicolumn{5}{|l|}{ Initial ADT (\%) } \\
\hline LHRH analog + NAs & $162(88.0)$ & $78(84.8)$ & $84(91.3)$ & \\
\hline LHRH analog & $11(6.0)$ & $7(7.6)$ & $4(4.3)$ & \\
\hline NAs & $7(3.8)$ & $5(5.4)$ & $2(2.2)$ & \\
\hline Others & $4(2.2)$ & $2(2.2)$ & $2(2.2)$ & 0.113 \\
\hline \multicolumn{5}{|l|}{ Mets at first line treatment (\%) } \\
\hline M0 & $57(31.0)$ & $27(29.4)$ & $30(32.6)$ & \\
\hline M1 & $127(69.0)$ & $65(70.7)$ & $62(67.4)$ & 0.345 \\
\hline \multicolumn{5}{|l|}{ Visceral mets at first line treatment $(\%)$} \\
\hline No & $165(89.7)$ & $82(89.1)$ & $83(90.2)$ & \\
\hline Yes & $19(10.3)$ & $10(10.9)$ & $9(9.8)$ & 0.809 \\
\hline \multicolumn{5}{|l|}{ LN mets at first line treatment $(\%)$} \\
\hline Non & $117(63.6)$ & $55(59.8)$ & $62(67.4)$ & \\
\hline Regional & $45(24.5)$ & $23(25.0)$ & $22(23.9)$ & \\
\hline Non-regional & $22(12.0)$ & $14(15.2)$ & $8(8.7)$ & 0.350 \\
\hline \multicolumn{5}{|l|}{ No. of bone mets at first line treatment (\%) } \\
\hline 0 & $83(45.1)$ & $40(43.5)$ & $43(46.7)$ & \\
\hline 1 & $23(12.5)$ & $11(12.0)$ & $12(13.0)$ & \\
\hline 2 & $15(8.2)$ & $8(8.7)$ & $7(7.6)$ & \\
\hline$>3$ & $63(34.2)$ & $33(35.9)$ & $30(32.6)$ & 0.948 \\
\hline \multicolumn{5}{|l|}{ ECOG-PS (\%) } \\
\hline 0 & $104(56.5)$ & $51(55.4)$ & $53(57.6)$ & \\
\hline 1 & $66(35.9)$ & $35(38.0)$ & $31(33.7)$ & \\
\hline$\geq 2$ & $14(7.6)$ & $6(6.6)$ & $8(8.7)$ & 0.684 \\
\hline $\begin{array}{l}\text { Neutrophil-lymphocyte ratio at first line treatment } \\
\qquad(\text { mean } \pm \text { SD) }\end{array}$ & $2.99 \pm 2.49$ & $3.08 \pm 2.93$ & $2.84 \pm 1.59$ & 0.648 \\
\hline $\mathrm{Hb}$ at first line treatment $(\mathrm{g} / \mathrm{dL})($ mean $\pm \mathrm{SD})$ & $12.2 \pm 1.8$ & $12.1 \pm 1.8$ & $12.3 \pm 1.8$ & 0.627 \\
\hline Platelet count at first line treatment $\left(10^{3} / \mathrm{uL}\right)($ mean $\pm \mathrm{SD})$ & $213 \pm 75$ & $215 \pm 80$ & $210 \pm 71$ & 0.640 \\
\hline ALP at first line treatment (U/L) (quartile) & $248(202,350)$ & $252(199,370)$ & $246(213,344)$ & 0.884 \\
\hline $\mathrm{LDH}$ at first line treatment (U/L) (quartile) & $200(182,238)$ & $201(177,231)$ & $200(185,240)$ & 0.675 \\
\hline Albumin (g/dL) (quartile) & $4.1(3.8,4.4)$ & $4.1(3.6,4.3)$ & $4.2(3.9,4.4)$ & 0.126 \\
\hline CRP (mg/dL) (quartile) & $0.1(0.05,0.32)$ & $0.1(0.05,0.23)$ & $0.1(0.05,0.48)$ & 0.670 \\
\hline
\end{tabular}

CRPC: castration-resistant prostate cancer, Abi: abiraterone, Enz: enzalutamide, SD: standard deviation, SRE: skeletal-related events, PSA: prostate-specific antigen, ADT: androgen deprivation therapy, LN: lymph node, Mets: metastasis, ECOG-PS: Eastern Cooperative Oncology Group performance status, Hb: hemoglobin, ALP: alkaline phosphatase, LDH: lactate dehydrogenase, CRP: C-reactive protein, LHRH: luteinizing hormone-releasing hormone, NAs: nonsteroidal antiandrogens. 

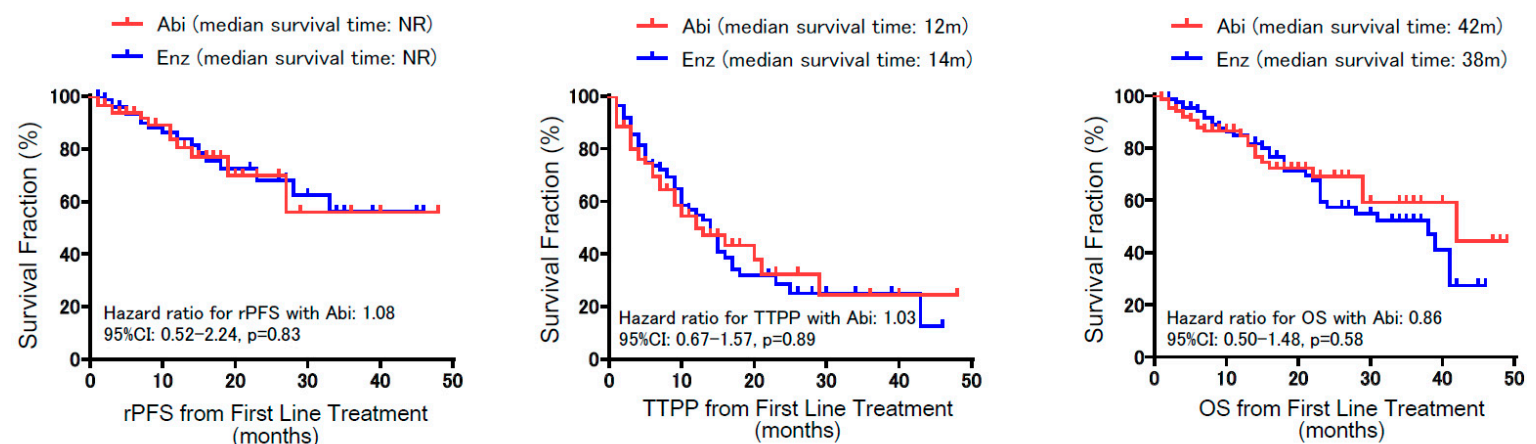

Figure 2. Kaplan-Meier curves for radiographic progression-free survival (rPFS), time to prostate specific antigen (PSA) progression (TTPP), and overall survival (OS) from the initiation of the first-line treatment. Note that there was no significant difference in rPFS, TTPP, and OS between abiraterone and enzalutamide from the first-line treatment.

Table 2. Patient characteristics in 84 CRPC patients treated with ASIs as 2nd treatment.

\begin{tabular}{|c|c|c|c|c|}
\hline Variables & Total $(n=84)$ & Abi to Enz $(n=46)$ & Enz to Abi $(n=38)$ & $p$ Value \\
\hline Age (mean \pm SD) & $73.0+7.9$ & $71.8+7.3$ & $74.4+8.4$ & ns \\
\hline \multicolumn{5}{|l|}{ SRE during follow-up } \\
\hline No $(\%)$ & $63(75.0)$ & $32(69.6)$ & $31(81.6)$ & \\
\hline Yes $(\%)$ & $21(25.0)$ & $14(30.4)$ & $7(18.4)$ & ns \\
\hline $\begin{array}{l}\text { Median PSA level at 2nd line treatment } \\
\text { (ng/mL) (quartile) }\end{array}$ & $21.3(3.8,93.2)$ & $21.5(8.1,61.3)$ & $21.0(3.4,94.3)$ & ns \\
\hline \multicolumn{5}{|l|}{ Gleason sum score $(\%)$} \\
\hline$\leq 7$ & $11(13.1)$ & $8(17.4)$ & $3(7.9)$ & \\
\hline 8 & $16(19.0)$ & $6(13.0)$ & $10(26.3)$ & \\
\hline 9 & $53(63.1)$ & $30(65.2)$ & $23(60.5)$ & \\
\hline 10 & $4(4.8)$ & $2(4.4)$ & $2(5.3)$ & ns \\
\hline \multicolumn{5}{|l|}{ Mets at 2nd line treatment (\%) } \\
\hline M0 & $16(19.0)$ & $9(19.6)$ & $7(18.4)$ & \\
\hline M1 & $68(81.0)$ & $37(80.4)$ & $31(81.6)$ & ns \\
\hline \multicolumn{5}{|l|}{ Visceral mets at 2nd line treatment (\%) } \\
\hline No & $75(88.2)$ & $39(84.8)$ & $36(94.7)$ & \\
\hline Yes & $9(11.8)$ & $7(15.2)$ & $2(5.3)$ & ns \\
\hline \multicolumn{5}{|l|}{ LN mets at 2nd line treatment (\%) } \\
\hline No & $59(70.2)$ & $31(67.4)$ & $28(73.7)$ & \\
\hline Yes & $25(29.8)$ & $15(32.6)$ & $10(26.3)$ & ns \\
\hline \multicolumn{5}{|l|}{ Bone mets at 2 nd line treatment ( $\%)$} \\
\hline No & $25(29.8)$ & $14(30.4)$ & $11(29.0)$ & \\
\hline Yes & $59(70.2)$ & $32(69.6)$ & $27(71.1)$ & ns \\
\hline \multicolumn{5}{|l|}{ Taxanes during follow-up } \\
\hline No & $72(85.7)$ & $41(89.1)$ & $30(79.0)$ & \\
\hline Yes & $12(14.3)$ & $5(10.9)$ & $8(21.0)$ & ns \\
\hline \multicolumn{5}{|l|}{ ECOG-PS (\%) } \\
\hline 0 & $34(40.5)$ & $17(37.0)$ & $17(44.7)$ & \\
\hline 1 & $45(53.6)$ & $27(58.7)$ & $18(47.4)$ & \\
\hline$\geq 2$ & $5(5.9)$ & $2(4.4)$ & $3(7.9)$ & ns \\
\hline \multicolumn{5}{|l|}{ PSA decline $\geq 50 \%$ at first line treatment } \\
\hline No $(\%)$ & $43(51.2)$ & $28(60.9)$ & $15(39.5)$ & \\
\hline Yes $(\%)$ & $41(48.8)$ & $18(39.1)$ & $23(60.5)$ & 0.037 \\
\hline
\end{tabular}

CRPC: castration-resistant prostate cancer, Abi: abiraterone, Enz: enzalutamide, SD: standard deviation, SRE: skeletal-related events, PSA: prostate-specific antigen, ADT: androgen deprivation therapy, LN: lymph node, Mets: metastasis, ECOG-PS: Eastern Cooperative Oncology Group performance status. 


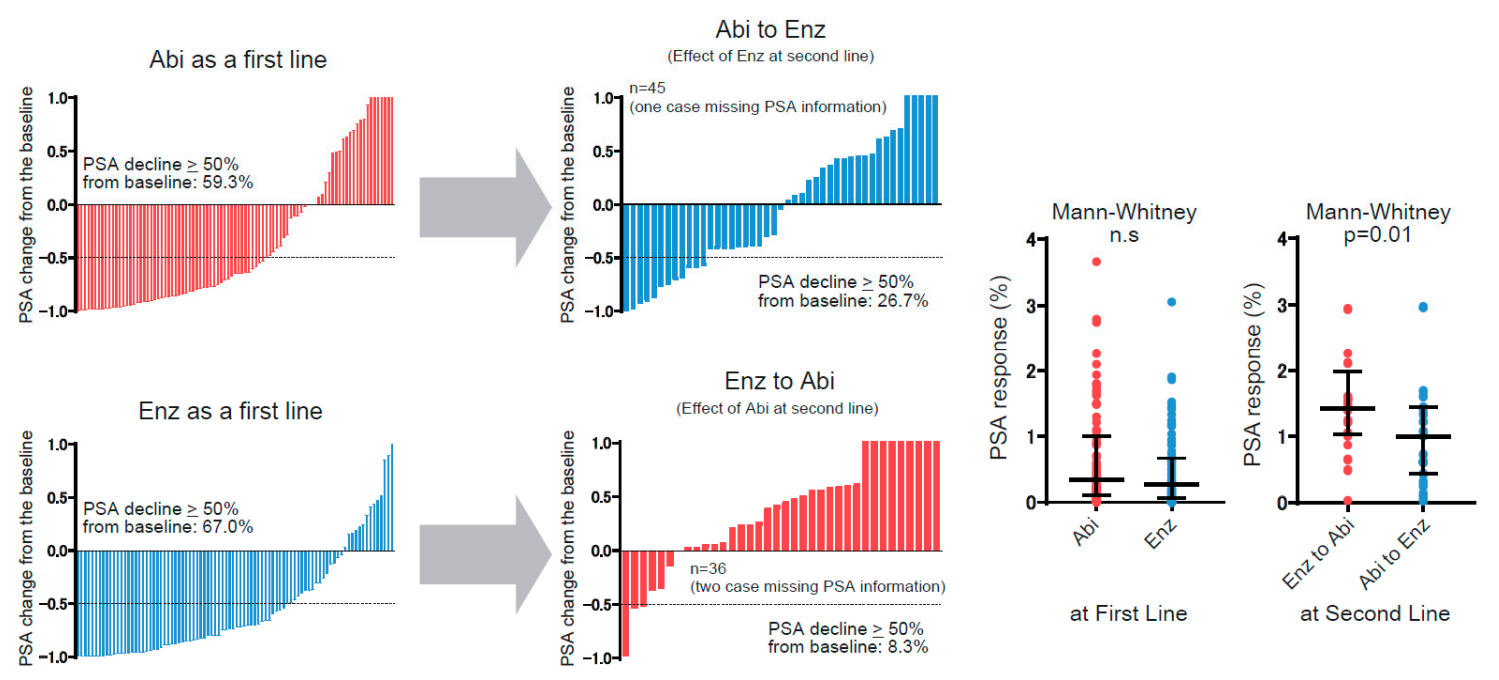

Figure 3. Waterfall plot illustrating the PSA response to first and second line androgen signaling inhibitors (ASIs). Dotted lines express the level of $>50 \%$ PSA decline. In the first-line treatment, PSA decline of more than $50 \%$ from the baseline is observed in $59.3 \%$ (54 of 92) for abiraterone and $67 \%$ (60 of 92) for enzalutamide, with no significant difference (Chi-square: $p=0.28$ ). In the second-line treatment, PSA decline of more than $50 \%$ from the initiation of second-line treatment is significantly less observed in abiraterone as a second line $(8.3 \%)$ compared with enzalutamide following abiraterone $(26.7 \%)$ (Chi-square: $p=0.03)$. PSA response from the baseline at each line is shown in the right panel.
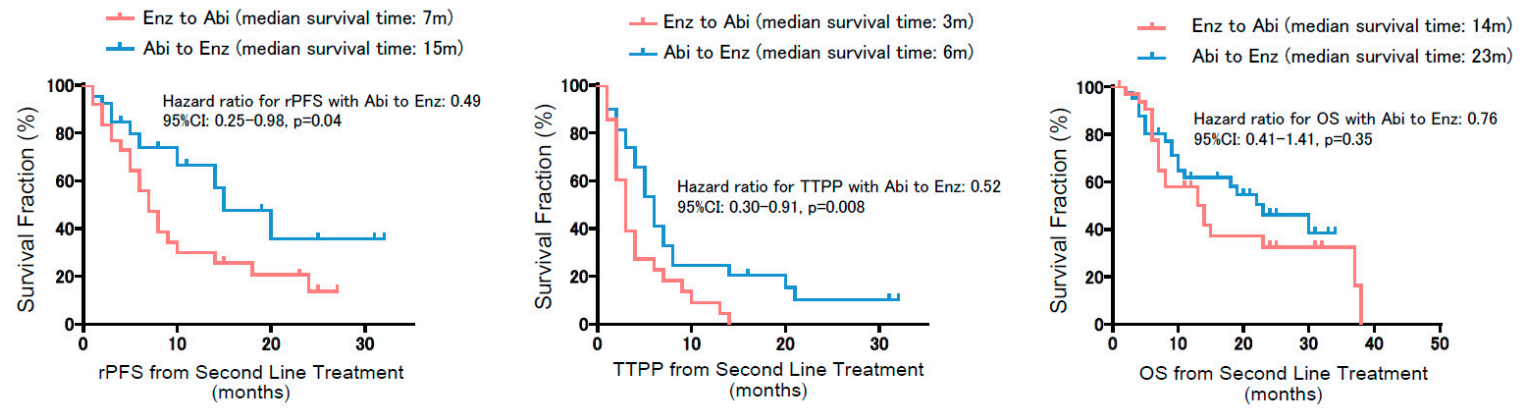

Figure 4. Kaplan-Meier curves for radiographic progression-free survival (rPFS), time to PSA progression (TTPP), and overall survival (OS) from the initiation of the second-line treatment. Note that rPFS and TTPP from the initiation of the second line significantly favored enzalutamide following abiraterone compared to vice versa.

Given that TTPP and rPFS for the treatment using abiraterone and enzalutamide from the time point of the first line appeared to be similar, whereas these drugs might offer distinct effects when applied a second line, we hypothesized that the extent of cross-resistance for these ASIs differs according to the first line. To assess the clinical impact of the order of sequential ASIs from the initiation of the second line, we performed multivariate analysis including putative variables for the prediction of TTPP and rPFS (Table 3). For the TTPP at the second line, there were several variables serving as an independent predictor of TTPP, including visceral metastasis at the initiation of second line (HR: 3.647, 95\%CI: 1.003-23.634, $p=0.049$ ), a PSA decline of $>50 \%$ during first-line treatment (HR: 0.641 , 95\%CI: 0.401-0.933, $p=0.038$ ), and ECOG-PS (HR: 2.154, 95\%CI: 1.163-4.154, $p=0.014$ ), as well as the treatment sequence (HR: 1.791, 95\%CI: 1.091-3.163, $p=0.043$ for 'enzalutamide to abiraterone'). For rPFS, visceral metastasis only remained an independent predictor for rPFS (HR: 3.647, 95\%CI: $1.182-19.278, p=0.032$ ). Finally, we assessed the correlation of rPFS and TTPP with OS from the initiation of second-line treatment (Table 4). The association with OS appeared robust in rPFS compared to TTPP (Spearman's correlation coefficients of 0.610 and 0.468 , respectively). 
Table 3. Multivariate analysis adjusting with putative variables for the prediction of TTPP and rPFS from the initiation of second line treatment.

\begin{tabular}{|c|c|c|c|c|c|c|c|c|}
\hline \multirow[b]{2}{*}{ Variables } & \multicolumn{4}{|c|}{ TTPP at 2nd Line } & \multicolumn{4}{|c|}{ Radiographic PFS } \\
\hline & HR & \multicolumn{2}{|c|}{$95 \%$ CI } & $p$ Value & HR & \multicolumn{2}{|c|}{$95 \% \mathrm{CI}$} & $p$ Value \\
\hline & & & & & & & & \\
\hline Abi to Enz & Ref & & & & Ref & & & \\
\hline Enz to $\mathrm{Abi}$ & 1.791 & 1.091 & 3.163 & 0.043 & 1.538 & 0.855 & 3.019 & 0.219 \\
\hline \multicolumn{9}{|c|}{$\begin{array}{l}\text { Visceral mets at 2nd line treatment } \\
\text { No }\end{array}$} \\
\hline Yes & 3.647 & 1.003 & 23.634 & 0.049 & 3.647 & 1.182 & 19.278 & 0.032 \\
\hline \multicolumn{9}{|c|}{$\begin{array}{l}\mathrm{LN} \text { mets at } 2 \mathrm{nd} \text { line } \\
\text { treatment }\end{array}$} \\
\hline No & Ref & & & & Ref & & & \\
\hline Yes & 1.663 & 0.847 & 3.406 & 0.141 & 1.233 & 0.784 & 2.392 & 0.221 \\
\hline \multicolumn{9}{|c|}{ Bone mets at 2 nd line treatment } \\
\hline No & Ref & & & & Ref & & & \\
\hline Yes & 1.946 & 0.972 & 4.071 & 0.06 & 1.392 & 0.872 & 4.281 & 0.099 \\
\hline \multicolumn{9}{|c|}{$\begin{array}{c}\text { PSA decline } \geq 50 \% \text { at first } \\
\text { line }\end{array}$} \\
\hline No & Ref & & & & Ref & & & \\
\hline Yes & 0.641 & 0.401 & 0.933 & 0.038 & 0.865 & 0.431 & 1.283 & 0.492 \\
\hline \multicolumn{9}{|l|}{ ECOG-PS } \\
\hline 0 & Ref & & & & Ref & & & \\
\hline$>1$ & 2.154 & 1.163 & 4.154 & 0.014 & 1.538 & 0.699 & 2.193 & 0.293 \\
\hline
\end{tabular}

TTPP: time to PSA progression, HR: hazard ratio, CI: confidential interval, ECOG-PS: Eastern Cooperative Oncology Group performance status

Table 4. Correlation of rPFS and TTPP with OS from the initiation of second line treatment.

\begin{tabular}{ccc}
\hline Variables & Spearman's Correlation Coefficient (SCC) (95\%CI) & $p$ Value \\
\hline rPFS & $0.601(0.411-0.722)$ & $<0.001$ \\
TTPP & $0.468(0.275-0.625)$ & $<0.001$ \\
\hline
\end{tabular}

rPFS: radiographic progression-free survival, TTPP: time to PSA progression, OS: overall survival, CI: confidence interval.

\section{Discussion}

In the present study, we investigated rPFS and PSA response in sequential treatment using ASIs for patients newly diagnosed with CRPC. Reducing the effect of confounding variables between treatment arms by propensity matching identified a pair-matched cohort of 184 patients with no significant differences among all variables between treatment arms, which allowed us to compare the treatment outcome for these ASIs with a minimally biased retrospective setting. Since recent studies have suggested that rPFS serves as a robust surrogate in the prediction of OS for treatment using abiraterone [30] and enzalutamide [29], we chose rPFS for both the first and subsequent second line as a primary endpoint as well as PSA response.

There have been a number of retrospective studies assessing sequential treatment using ASIs (i.e., 'abiraterone following enzalutamide' [18-21,23,25,26] and 'enzalutamide following abiraterone' $[14-17,20,22-26])$. In terms of PSA response, the results from those studies seemed to favor 'enzalutamide following abiraterone' [38], whereas no report has demonstrated an improved OS in this treatment sequence. A phase 4 multi-institutional, single-arm, open-label study reported by de Bono et al. enrolled 214 CRPC patients with progressions after $\geq 24$ weeks of abiraterone treatment, and rPFS for the subsequent enzalutamide was examined as the primary endpoint [27]. The median duration of enzalutamide treatment for the second line was 5.7 months, and the median rPFS was 8.1 months. In their study, 69 out of 214 (32.2\%) deceased during follow-up, and 69 (32.2\%) patients were previously treated with chemotherapy before enzalutamide. In the present study, 55 out of 
$184(29.9 \%)$ patients deceased during follow-up, and the median rPFS from the initiation of the second line for 'enzalutamide following abiraterone' was 15 months, which appeared to be longer than their study [27]. We assume this could be at least in large part due to the fact that the present study included M0 patients at the initiation of the second line (19\%), and all the patients were chemotherapy-naïve.

In regard to the PSA response, the phase 2 randomized cross-over trial exhibited that a PSA decline of $\geq 50 \%$ for first-line treatment was $53 \%$ in abiraterone versus $73 \%$ in enzalutamide $(p=0.004)$, whereas median TTPP was similar (7.4 months in abiraterone vs 8.0 months in enzalutamide: HR $=0.88$, 95\%CI: 0.61-1.27) [39]. Although there was a similar trend of better PSA response in enzalutamide for the first line, the present study did not show a significant difference in either the achievement of PSA decline $\geq 50 \%$ or PSA response between these ASIs as for the first line (Figure 3). TTPP for the first line also showed no difference between treatment arms. Recently, updated results from a phase 2 study were reported by Khalaf et al. [28]. They showed that PSA response rate and TTPP were better for second-line enzalutamide following abiraterone compared with the reverse, and treatment arm was an independent predictor of PSA progression during second-line treatment. Consistent with their results, the present study also exhibited better PSA response and TTPP in patients treated with enzalutamide as a second line following abiraterone. These data indicate that, although the effect as a first line is similar between these ASIs, the extent of cross-resistance might differ towards less resistance to enzalutamide following abiraterone. The results showed no difference in rPFS and TTPP between ASIs as the first-line treatment, whereas rPFS and TTPP from the initiation of the second line were significantly longer in enzalutamide following abiraterone. Furthermore, we examined an association between rPFS/TTPP and OS from the initiation of second-line ASIs, as shown in Table 4 . As expected, both rPFS and TTPP were significantly correlated with OS $(p<0.001$ in both rPFS and TTPP). Importantly, higher SCC in rPFS was observed compared to that in TTPP (0.601 in rPFS vs 0.468 in TTPP), indicating rPFS is a robust surrogate for predicting OS, which is consistent with the previous report by Lorente et al. [31].

The limitations in the present study involved its retrospective design and relatively small sample size, as well as a lack of consideration of biomarkers such as ARV7 [40]. In addition, the findings in the current study were still subject to selection bias, which we sought to address by using a propensity score-matching model to approximate random assignment. Residual unmeasured confounding factors may have affected the clinical impact of ASIs observed in the study. Finally, multivariate analysis in the current study did not demonstrate treatment sequence and ECOG-PS as independent predictors for predicting rPFS from the initiation of the second-line treatment, which could be due to the low statistical power. To clarify the best treatment strategy for individual patients, further prospective work is warranted.

\section{Conclusions}

We compared rPFS and PSA response in sequential treatment using abiraterone and enzalutamide for newly diagnosed CRPC patients in a retrospective paired-matched cohort by propensity score matching. For the first line, rPFS and TTPP were both similar between ASIs. For the second line, enzalutamide following abiraterone was likely to achieve longer rPFS and TTPP compared to abiraterone following enzalutamide. These findings could allow physicians to select the optimal treatment sequence using ASIs in daily practice.

Author Contributions: Conceptualization, K.K., Y.F. and T.U.; Methodology, K.K., K.S. and N.T.; Software, K.K., T.M. and A.I.; Validation, K.K., T.T. (Takeshi Tsutsumi) and T.T. (Takuya Tsujino); Formal Analysis K.K., Y.Y. and Y.N; Investigation, K.K., T.T. (Tomoaki Takai) and K.M.; Resources, K.T., T.T. (Tomohito Tanaka) and H.U.; Data Curation, K.K., H.H. and H.N.; Writing-Original Draft Preparation, K.K., N.I. and K.T.; Writing-Review \& Editing, K.K., T.I. and H.A.; Visualization, K.K. and U.F.; Supervision, T.I. and H.A.; Project Administration, K.K. and H.A.; Funding Acquisition, K.K. and H.A.

Funding: This work was partially supported by the Foundation of Research Basis Development in Osaka Medical College (Japan), Grant-in-Aid No. 19K18624 (Japan Society for the Promotion of Science: JSPS), the Uehara Memorial Foundation, the NOVARTIS Foundation (Japan) for the Promotion of Science, Japan Research 
Foundation for Clinical Pharmacology, Yamaguchi Endocrine Disease Research Foundation, and the Takeda Science Foundation 2018 in Japan.

Conflicts of Interest: The authors have no conflict of interest.

$\begin{array}{ll}\text { Abbreviations } \\ \text { rPFS } & \begin{array}{l}\text { radiographic progression-free survival } \\ \text { overall survival }\end{array} \\ \text { OS } & \text { castration-resistant prostate cancer } \\ \text { CRPC } & \text { androgen signaling inhibitors } \\ \text { ASIs } & \text { time to PSA progression } \\ \text { TTPP } & \text { androgen deprivation therapy } \\ \text { ADT } & \text { randomized control trial } \\ \text { RCT } & \text { Spearman's correlation coefficient }\end{array}$

\section{References}

1. Siegel, R.L.; Miller, K.D.; Jemal, A. Cancer statistics, 2018. CA Cancer J. Clin. 2018, 68, 7-30. [CrossRef] [PubMed]

2. Mazzu, Y.Z.; Armenia, J.; Chakraborty, G.; Yoshikawa, Y.; Coggins, S.; Nandakumar, S.; Gerke, T.; Pomerantz, M.; Qiu, X.; Zhao, H.; et al. A novel mechanism driving poor-prognosis prostate cancer: Overexpression of the DNA repair gene, ribonucleotide reductase small subunit M2 (RRM2). Clin. Cancer Res. 2019, 25, 4480-4492. [CrossRef] [PubMed]

3. Sun, T.; Du, S.Y.; Armenia, J.; Qu, F.; Fan, J.; Wang, X.; Fei, T.; Komura, K.; Liu, S.X.; Lee, G.M.; et al. Expression of lncRNA MIR222HG co-transcribed from the miR-221/222 gene promoter facilitates the development of castration-resistant prostate cancer. Oncogenesis 2018, 7, 30. [CrossRef] [PubMed]

4. Komura, K.; Yoshikawa, Y.; Shimamura, T.; Chakraborty, G.; Gerke, T.A.; Hinohara, K.; Chadalavada, K.; Jeong, S.H.; Armenia, J.; Du, S.Y.; et al. ATR inhibition controls aggressive prostate tumors deficient in Y-linked histone demethylase KDM5D. J. Clin. Invest. 2018, 128, 2979-2995. [CrossRef] [PubMed]

5. Komura, K.; Jeong, S.H.; Hinohara, K.; Qu, F.; Wang, X.; Hiraki, M.; Azuma, H.; Lee, G.S.; Kantoff, P.W.; Sweeney, C.J. Resistance to docetaxel in prostate cancer is associated with androgen receptor activation and loss of KDM5D expression. Proc. Natl. Acad. Sci. USA 2016, 113, 6259-6264. [CrossRef] [PubMed]

6. De Bono, J.S.; Logothetis, C.J.; Molina, A.; Fizazi, K.; North, S.; Chu, L.; Chi, K.N.; Jones, R.J.; Goodman, O.B., Jr.; Saad, F.; et al. Abiraterone and increased survival in metastatic prostate cancer. N. Engl. J. Med. 2011, 364, 1995-2005. [CrossRef] [PubMed]

7. Scher, H.I.; Fizazi, K.; Saad, F.; Taplin, M.E.; Sternberg, C.N.; Miller, K.; de Wit, R.; Mulders, P.; Chi, K.N.; Shore, N.D.; et al. Increased survival with enzalutamide in prostate cancer after chemotherapy. N. Engl. J. Med. 2012, 367, 1187-1197. [CrossRef] [PubMed]

8. Beer, T.M.; Armstrong, A.J.; Rathkopf, D.E.; Loriot, Y.; Sternberg, C.N.; Higano, C.S.; Iversen, P.; Bhattacharya, S.; Carles, J.; Chowdhury, S.; et al. Enzalutamide in metastatic prostate cancer before chemotherapy. N. Engl. J. Med. 2014, 371, 424-433. [CrossRef]

9. Ryan, C.J.; Smith, M.R.; de Bono, J.S.; Molina, A.; Logothetis, C.J.; de Souza, P.; Fizazi, K.; Mainwaring, P.; Piulats, J.M.; $\mathrm{Ng}$, S.; et al. Abiraterone in metastatic prostate cancer without previous chemotherapy. N. Engl. J. Med. 2013, 368, 138-148. [CrossRef]

10. Fizazi, K.; Tran, N.; Fein, L.; Matsubara, N.; Rodriguez-Antolin, A.; Alekseev, B.Y.; Ozguroglu, M.; Ye, D.; Feyerabend, S.; Protheroe, A.; et al. Abiraterone plus Prednisone in Metastatic, Castration-Sensitive Prostate Cancer. N. Engl. J. Med. 2017, 377, 352-360. [CrossRef]

11. James, N.D.; de Bono, J.S.; Spears, M.R.; Clarke, N.W.; Mason, M.D.; Dearnaley, D.P.; Ritchie, A.W.S.; Amos, C.L.; Gilson, C.; Jones, R.J.; et al. Abiraterone for Prostate Cancer Not Previously Treated with Hormone Therapy. N. Engl. J. Med. 2017, 377, 338-351. [CrossRef] [PubMed]

12. Gillessen, S.; Attard, G.; Beer, T.M.; Beltran, H.; Bossi, A.; Bristow, R.; Carver, B.; Castellano, D.; Chung, B.H.; Clarke, N.; et al. Management of Patients with Advanced Prostate Cancer: The Report of the Advanced Prostate Cancer Consensus Conference APCCC 2017. Eur. Urol. 2017, 73, 178-211. [CrossRef] [PubMed] 
13. Chiong, E.; Murphy, D.G.; Akaza, H.; Buchan, N.C.; Chung, B.H.; Kanesvaran, R.; Khochikar, M.; Letran, J.; Lojanapiwat, B.; Ng, C.F.; et al. Management of patients with advanced prostate cancer in the Asia Pacific region: 'real-world' consideration of results from the Advanced Prostate Cancer Consensus Conference (APCCC) 2017. BJU Int. 2019, 123, 22-34. [CrossRef] [PubMed]

14. Azad, A.A.; Eigl, B.J.; Murray, R.N.; Kollmannsberger, C.; Chi, K.N. Efficacy of enzalutamide following abiraterone acetate in chemotherapy-naive metastatic castration-resistant prostate cancer patients. Eur. Urol. 2015, 67, 23-29. [CrossRef] [PubMed]

15. Badrising, S.; van der Noort, V.; van Oort, I.M.; van den Berg, H.P.; Los, M.; Hamberg, P.; Coenen, J.L.; van den Eertwegh, A.J.; de Jong, I.J.; Kerver, E.D.; et al. Clinical activity and tolerability of enzalutamide (MDV3100) in patients with metastatic, castration-resistant prostate cancer who progress after docetaxel and abiraterone treatment. Cancer 2014, 120, 968-975. [CrossRef] [PubMed]

16. Brasso, K.; Thomsen, F.B.; Schrader, A.J.; Schmid, S.C.; Lorente, D.; Retz, M.; Merseburger, A.S.; von Klot, C.A.; Boegemann, M.; de Bono, J. Enzalutamide Antitumour Activity Against Metastatic Castration-resistant Prostate Cancer Previously Treated with Docetaxel and Abiraterone: A Multicentre Analysis. Eur. Urol. 2015, 68, 317-324. [CrossRef] [PubMed]

17. David, T.; Natalie, C.; Omi, P. Enzalutamide after failure of docetaxel and abiraterone in metastatic castrate resistant prostate cancer (mCRPC): Results from an expanded access program. J. Clin. Oncol. 2014, $32,188$.

18. Loriot, Y.; Bianchini, D.; Ileana, E.; Sandhu, S.; Patrikidou, A.; Pezaro, C.; Albiges, L.; Attard, G.; Fizazi, K.; De Bono, J.S.; et al. Antitumour activity of abiraterone acetate against metastatic castration-resistant prostate cancer progressing after docetaxel and enzalutamide (MDV3100). Ann. Oncol. 2013, 24, 1807-1812. [CrossRef] [PubMed]

19. Maughan, B.L.; Luber, B.; Nadal, R.; Antonarakis, E.S. Comparing Sequencing of Abiraterone and Enzalutamide in Men with Metastatic Castration-Resistant Prostate Cancer: A Retrospective Study. Prostate 2017, 77, 33-40. [CrossRef]

20. Mori, K.; Kimura, T.; Onuma, H.; Kimura, S.; Yamamoto, T.; Sasaki, H.; Miki, J.; Miki, K.; Egawa, S. Lactate dehydrogenase predicts combined progression-free survival after sequential therapy with abiraterone and enzalutamide for patients with castration-resistant prostate cancer. Prostate 2017, 77, 1144-1150. [CrossRef]

21. Noonan, K.L.; North, S.; Bitting, R.L.; Armstrong, A.J.; Ellard, S.L.; Chi, K.N. Clinical activity of abiraterone acetate in patients with metastatic castration-resistant prostate cancer progressing after enzalutamide. Ann. Oncol. 2013, 24, 1802-1807. [CrossRef] [PubMed]

22. Schrader, A.J.; Boegemann, M.; Ohlmann, C.H.; Schnoeller, T.J.; Krabbe, L.M.; Hajili, T.; Jentzmik, F.; Stoeckle, M.; Schrader, M.; Herrmann, E.; et al. Enzalutamide in castration-resistant prostate cancer patients progressing after docetaxel and abiraterone. Eur. Urol. 2014, 65, 30-36. [CrossRef] [PubMed]

23. Terada, N.; Maughan, B.L.; Akamatsu, S.; Kobayashi, T.; Yamasaki, T.; Inoue, T.; Kamba, T.; Ogawa, O.; Antonarakis, E.S. Exploring the optimal sequence of abiraterone and enzalutamide in patients with chemotherapy-naive castration-resistant prostate cancer: The Kyoto-Baltimore collaboration. Int. J. Urol. 2017, 24, 441-448. [CrossRef] [PubMed]

24. Thomsen, F.B.; Roder, M.A.; Rathenborg, P.; Brasso, K.; Borre, M.; Iversen, P. Enzalutamide treatment in patients with metastatic castration-resistant prostate cancer progressing after chemotherapy and abiraterone acetate. Scand. J. Urol. 2014, 48, 268-275. [CrossRef] [PubMed]

25. Matsubara, N.; Yamada, Y.; Tabata, K.I.; Satoh, T.; Kamiya, N.; Suzuki, H.; Kawahara, T.; Uemura, H.; Yano, A.; Kawakami, S.; et al. Abiraterone Followed by Enzalutamide Versus Enzalutamide Followed by Abiraterone in Chemotherapy-naive Patients with Metastatic Castration-resistant Prostate Cancer. Clin. Genitourin. Cancer 2018, 16, 142-148. [CrossRef] [PubMed]

26. Miyake,H.; Hara, T.; Tamura, K.; Sugiyama, T.; Furuse,H.; Ozono, S.; Fujisawa, M. Comparative Assessment of Efficacies Between 2 Alternative Therapeutic Sequences with Novel Androgen Receptor-Axis-Targeted Agents in Patients with Chemotherapy-Naive Metastatic Castration-Resistant Prostate Cancer. Clin. Genitourin. Cancer 2017, 15, e591-e597. [CrossRef] [PubMed]

27. De Bono, J.S.; Chowdhury, S.; Feyerabend, S.; Elliott, T.; Grande, E.; Melhem-Bertrandt, A.; Baron, B.; Hirmand, M.; Werbrouck, P.; Fizazi, K. Antitumour Activity and Safety of Enzalutamide in Patients with Metastatic Castration-resistant Prostate Cancer Previously Treated with Abiraterone Acetate Plus Prednisone for $\geq 24$ weeks in Europe. Eur. Urol. 2018, 74, 37-45. [CrossRef] 
28. Khalaf, D.; Annala, M.; Finch, D.L.; Oja, C.D.; Vergidis, J.; Zulfiqar, M.; Sunderland, K.; Beja, K.; Vandekerkhove, G.R.; Gleave, M.; et al. Phase 2 randomized cross-over trial of abiraterone + prednisone $(\mathrm{ABI}+\mathrm{P})$ vs. enzalutamide (ENZ) for patients (pts) with metastatic castration resistant prostate cancer (mCPRC): Results for 2nd-line therapy. J. Clin. Oncol. 2018, 36, 5015. [CrossRef]

29. Rathkopf, D.E.; Beer, T.M.; Loriot, Y.; Higano, C.S.; Armstrong, A.J.; Sternberg, C.N.; de Bono, J.S.; Tombal, B.; Parli, T.; Bhattacharya, S.; et al. Radiographic Progression-Free Survival as a Clinically Meaningful End Point in Metastatic Castration-Resistant Prostate Cancer: The PREVAIL Randomized Clinical Trial. JAMA Oncol. 2018, 4, 694-701. [CrossRef]

30. Morris, M.J.; Molina, A.; Small, E.J.; de Bono, J.S.; Logothetis, C.J.; Fizazi, K.; de Souza, P.; Kantoff, P.W.; Higano, C.S.; Li, J.; et al. Radiographic progression-free survival as a response biomarker in metastatic castration-resistant prostate cancer: COU-AA-302 results. J. Clin. Oncol. 2015, 33, 1356-1363. [CrossRef]

31. Lorente, D.; Castro, E.; Lozano, R.; Puente, J.; Romero-Laorden, N.; Morales-Barrera, R.; Rodriguez Vida, A.; Sáez, M.I.; Mendez-Vidal, M.J.; Fernandez, E.; et al. Correlation between time to PSA progression (TTPP), radiographic progression-free survival (rPFS) and overall survival (OS) in first-line abiraterone/enzalutamide (Abi/Enza) and docetaxel (Doc) treated patients in a prospective cohort study. J. Clin. Oncol. 2019, 37, 267. [CrossRef]

32. World Medical, A. World Medical Association Declaration of Helsinki: Ethical principles for medical research involving human subjects. JAMA 2013, 310, 2191-2194.

33. Scher, H.I.; Halabi, S.; Tannock, I.; Morris, M.; Sternberg, C.N.; Carducci, M.A.; Eisenberger, M.A.; Higano, C.; Bubley, G.J.; Dreicer, R.; et al. Design and end points of clinical trials for patients with progressive prostate cancer and castrate levels of testosterone: Recommendations of the Prostate Cancer Clinical Trials Working Group. J. Clin Oncol. 2008, 26, 1148-1159. [CrossRef]

34. Hussain, M.; Wolf, M.; Marshall, E.; Crawford, E.D.; Eisenberger, M. Effects of continued androgen-deprivation therapy and other prognostic factors on response and survival in phase II chemotherapy trials for hormone-refractory prostate cancer: A Southwest Oncology Group report. J. Clin. Oncol. 1994, 12, 1868-1875. [CrossRef]

35. Taylor, C.D.; Elson, P.; Trump, D.L. Importance of continued testicular suppression in hormone-refractory prostate cancer. J. Clin. Oncol. 1993, 11, 2167-2172. [CrossRef]

36. Eisenhauer, E.A.; Therasse, P.; Bogaerts, J.; Schwartz, L.H.; Sargent, D.; Ford, R.; Dancey, J.; Arbuck, S.; Gwyther, S.; Mooney, M.; et al. New response evaluation criteria in solid tumours: Revised RECIST guideline (version 1.1). Eur. J. Cancer 2009, 45, 228-247. [CrossRef]

37. Ryan, C.J.; Shah, S.; Efstathiou, E.; Smith, M.R.; Taplin, M.E.; Bubley, G.J.; Logothetis, C.J.; Kheoh, T.; Kilian, C.; Haqq, C.M.; et al. Phase II study of abiraterone acetate in chemotherapy-naive metastatic castration-resistant prostate cancer displaying bone flare discordant with serologic response. Clin. Cancer Res. 2011, 17, 4854-4861. [CrossRef]

38. Komura, K.; Sweeney, C.J.; Inamoto, T.; Ibuki, N.; Azuma, H.; Kantoff, P.W. Current treatment strategies for advanced prostate cancer. Int. J. Urol. 2017, 25, 220-231. [CrossRef]

39. Chi, K.N.; Annala, M.; Sunderland, K.; Khalaf, D.; Finch, D.; Oja, C.D.; Vergidis, J.; Zulfiqar, M.; Beja, K.; Vandekerkhove, G.; et al. A randomized phase II cross-over study of abiraterone + prednisone (ABI) vs. enzalutamide (ENZ) for patients (pts) with metastatic, castration-resistant prostate cancer (mCRPC). J. Clin. Oncol. 2017, 35, 5002. [CrossRef]

40. Qu, F.; Xie, W.; Nakabayashi, M.; Zhang, H.; Jeong, S.H.; Wang, X.; Komura, K.; Sweeney, C.J.; Sartor, O.; Lee, G.M.; et al. Association of AR-V7 and Prostate-Specific Antigen RNA Levels in Blood with Efficacy of Abiraterone Acetate and Enzalutamide Treatment in Men with Prostate Cancer. Clin. Cancer Res. 2017, 23, 726-734. [CrossRef]

(C) 2019 by the authors. Licensee MDPI, Basel, Switzerland. This article is an open access article distributed under the terms and conditions of the Creative Commons Attribution (CC BY) license (http://creativecommons.org/licenses/by/4.0/). 